\title{
MODELOS EDUCATIVOS Y TENDENCIAS PEDAGÓGICAS: LA PEDAGOGÍA DEL AMOR ${ }^{1}$
}

\section{EDUCATIONAL MODELS AND PEDAGOGICAL TRENDS: THE PEDAGOGY OF LOVE}

\section{MODELOS EDUCATIVOS E TENDÊNCIAS PEDAGÓGICAS: A PEDAGOGIA DO AMOR}

\section{Alexander Ortiz Ocaña ${ }^{2}$ \\ Universidad del Magdalena}

\section{RESUMEN}

El concepto de pedagogía, el tema pedagógico y la pedagogía como ciencia, disciplina, saber o reflexión, han sido estudiados por múltiples autores en todos los continentes. Destacan en este abordaje las tradiciones de Alemania, Francia, Estados Unidos y Latinoamérica. Las concepciones sobre la pedagogía que se han venido configurando a lo largo de $1 \quad$ Resultado del proyecto Escenarios formativos mediadores de la biopraxis de niños y niñas en contexto de pobreza, financiado por la Universidad del Magdalena. Santa Marta. Colombia. Grupo GIEDU: Grupo de Investigación en Infancia y Educación.

2 Docente de planta de tiempo completo de la Universidad del Magdalena, Santa Marta, Colombia. Doctor en Ciencias de la Educación. Profesor del Doctorado en Ciencias de la Educación RUDECOLOMBIA-Universidad del Magdalena, Colombia. E-mail: alexanderortiz2009@gmail. com / orcid.org/0000-0001-5594-9422 la historia de la educación difieren en las diversas regiones y países. Es por ello que un análisis minucioso, detallado y profundo de la configuración epistémica de la noción de pedagogía requiere tener en cuenta no sólo la propia dimensión epistémica de la misma sino además su dimensión geográfica. En este artículo se describen los modelos educativos contemporáneos, se hace una caracterización de las tendencias pedagógicas postmodernas, y se reflexiona sobre el amor y el amar como modelo pedagógico, introduciendo la denominación Pedagogía del Amor. 


\section{PALABRAS CLAVE}

Educación, formación, pedagogía, modelo pedagógico, tendencia pedagógica, pedagogía del amor.

\section{ABSTRACT}

The concept of pedagogy, pedagogical subject and the pedagogy as a science, discipline, knowledge and reflection, have been studied by many authors on all continents. This approach include the traditions of Germany, France, United States and Latin America. The conceptions of pedagogy have been set up throughout the history of education differ in the various regions and countries. This is why a thorough, detailed and profound analysis of the notion of pedagogy epistemic configuration requires to take into account not only the own epistemic from the same dimension but also its geographical dimension. This article describes the contemporary educational models, a characterization of postmodern educational trends is made, and it reflects on love and loving as a pedagogic model, introducing the term pedagogy of love.

\section{KEY WORDS}

Education, training, pedagogy, pedagogical model, educational trend, pedagogy of love.

\section{RESUMO}

O conceito de pedagogia, assunto pedagógico e a pedagogia como ciência, disciplina, conhecimento e reflexão, têm sido estudados por muitos autores em todos os continentes. Esta abordagem incluem as tradições da Alemanha, França, Estados Unidos e América Latina. As concepções da pedagogia foram criadas ao longo da história da educação são diferentes nas várias regiões e países. Portanto, uma análise minuciosa, detalhada e profunda da noção de configuração epistêmica de pedagogia requer para levar em conta não só o própria epistêmica da mesma dimensão, mas também sua dimensão geográfica. Este artigo descreve os modelos educacionais contemporâneos, é feita uma caracterização das tendências educacionais pós-moderno, e isso reflete em amar e amar como um modelo pedagógico, introduzindo a pedagogia termo de amor.

\section{PALAVRAS-CHAVE:}

educação, formação, pedagogia, tendência educacional, modelo pedagógico, pedagogia do amor.

\section{INTRODUCCIÓN}

La complejidad de la educación como fenómeno sociocultural exige la participación de múltiples ciencias y disciplinas para su estudio amplio y profundo, y así poder comprender sus diversas aristas, contextos y dimensiones. De esta manera, la filosofía, la psicología, la antropología, la sociología, y las neurociencias, entre otras, se convierten en disciplinas educativas, que no sólo estudian su objeto propio sino además el campo de la educación. Muchas de estas ciencias y disciplinas científicas han sido canales epistémicos para la configuración de teorías educativas a finales del siglo $X X$ y principios del siglo XXI. La progresiva incorporación al discurso educativo de muchas de sus nociones y conceptos ha acrecentado el interés actual por el discurso como tecnología que produce subjetividades (Herrera, 2013).

Las ciencias de la educación están inmersas en una revolución epistémica, epistemológica e incluso ontológica. La pedagogía se desarrolla a pasos agigantados, se amplia y diversifica, cada vez más se nutre de otras ciencias como la psicología, la sociología, la antropología, e incluso las neurociencias, cuyos hallazgos en los últimos 30 ó 40 años no deben ignorarse ni soslayarse en cualquier debate y reflexión pedagógica que se haga en las instituciones 
educativas. Los profesores no debemos hacer reverencia y mucho menos rechazo a los aportes teóricos que nos llegan de todas estas ciencias, más bien debemos integrarlos de manera armónica, coherente y creativa en nuestras prácticas pedagógicas cotidianas y en el modelo pedagógico de la organización educativa. Sin embargo, es evidente que los hallazgos de las diversas ciencias socio-humanas se incorporan con una lentitud extraordinaria a la pedagogía, y con mayor lentitud aún al saber pedagógico y a las prácticas pedagógicas de los profesores.

Dos conceptos importantes que los profesores debemos tener en cuenta en nuestra reflexión sobre los fundamentos epistémicos de nuestro modelo pedagógico de la organización educativa, son las nociones de practica pedagógica y saber pedagógico, introducidos por Zuluaga (2005) sustentándose en la obra de Foucault.

Las prácticas pedagógicas se refieren a las prácticas de enseñanza en diferentes espacios sociales mediante elementos del saber pedagógico (Zuluaga, 1987), incluyen además los modelos pedagógicos teóricos y prácticos que utilizan los profesores, algunos conceptos de diversas disciplinas que son asumidos por la pedagogía, los discursos pedagógicos institucionales (Zuluaga, 2005). En cambio el saber pedagógico (Zuluaga, 2001) se refiere a los elementos doxáticos configurados por las opiniones, normas, percepciones, tradiciones y conceptos sobre la escuela, la enseñanza, el aprendizaje, el profesor, la instrucción, la educación y la formación del estudiante. El saber pedagógico no es una teoría que sustituya - complemente las teorías pedagógicas, curriculares y didácticas que han proliferado a lo largo de la historia de la educación, es más bien "un concepto metodológico para reunir discursos a propósito de la enseñanza y la educación; es por tanto un conjunto de elementos dispares" (Zuluaga, 2005, p. 24).
El arte de enseñar requiere no sólo el domino del contenido científico y técnico por parte de profesor, sino que es necesario además, y quizá lo más importante un saber especializado tapizado por lo educativo. Para enseñar, no basta con saber la asignatura, nos dicen Hernández y Sancho (1989), título atinado de uno de sus libros, en el que se precisa que los profesores debemos tener conocimientos pedagógicos y habilidades curriculares para poder enseñar con sentido y de manera significativa, y que los conocimientos del área son insuficientes. "Una cosa es saber, otra saber enseñar, otra muy distinta saber despertar deseos de aprender y finalmente tener conciencia de que hay que seguir aprendiendo" (Santos, 2012, p. 66).

Para desarrollar una labor excelente en este punto del modelo pedagógico de la organización educativa es importante que los profesores lean y reflexionen no sólo sobre las propuestas educativas y pedagógicas más actuales configuradas en nuestro país sino a nivel internacional, y es importante además tener en cuenta aportes educativos que han emergido desde distintas disciplinas. Por ejemplo, sin lugar a dudas, Gregory Bateson fue uno de los pensadores más excepcionales, originales y creativos del siglo pasado, no sólo de la antropología sino de las ciencias humanas y sociales en general. Una de las aportaciones más significativas de Bateson (1987) es la distinción de tres niveles de educación. El primer nivel de aprendizaje lo denominó aprendizaje cero, y está relacionado con la simple transmisión de información, en la que juega un papel significativo la memoria. El segundo nivel de aprendizaje es el denominado deuteroaprendizaje, que está relacionado con la configuración cognitiva, con el fin de abstraer e incorporar la información adquirida. $\mathrm{Y}$ el tercer nivel de aprendizaje consiste en enseñar al estudiante a aprender, es decir, enseñar aptitudes y competencias para reconfigurar y volver a configurar su propio aprendizaje, 
de manera que el estudiante pueda atomizar y fragmentar, para luego volver a organizar su configuración cognitiva, es el aprender a aprender, es un aprendizaje configurativo, que dura toda la vida y que le permite al estudiante estar en constante cambio y transformación, reaprender y reconfigurarse todo el tiempo, incluso abandonando principios y nociones que configuran su intelecto y su comportamiento.

Flórez (2005) puntualiza que toda teoría pedagógica trata de responder de manera sistemática y coherente al menos estas preguntas: ¿qué tipo de ser humano se quiere formar? ¿Con qué experiencias se desarrolla un ser humano? ¿Quién debe dirigir el proceso educativo? ¿Con qué métodos y técnicas? Cada uno de estos interrogantes puede ser afrontado por alguna ciencia o disciplina: antropología, sociología, currículo, didáctica; pero sólo la pedagogía puede abordarlas de una manera holística, integrada y transdisciplinaria, dando origen así a los modelos teóricos en educación.

\section{MODELOS EDUCATIVOS CONTEMPORÁNEOS}

A lo largo de la historia de la educación se han configurado diversos modelos pedagógicos como representaciones ideales del mundo educativo real, que permiten explicar, comprender y transformar la práctica pedagógica de los profesores. Los modelos pedagógicos son dinámicos, están en constante cambio y transformación, sin embargo, en determinado momento pueden ser visualizados para aplicarlos al proceso formativo de una institución educativa determinada. El modelo pedagógico a nivel societal se configura a partir del ideal de ser humano que concibe la sociedad, para su implementación ésta organiza un sistema educativo que debe ser cumplido en las instituciones escolares.

Tradicionalmente se han clasificado los modelos pedagógicos usando el recurso antinómico.
De esta manera, desde una mirada dual y demasiado generalista, se ofrecen dos modelos pedagógicos antagónicos: la escuela nueva o pedagogía activa en contraposición con la escuela pasiva o pedagogía tradicional. Sin embargo, al observar las prácticas pedagógicas de los profesores podemos constatar otras corrientes, tendencias y enfoques pedagógicos. No es posible entonces esbozar sólo estas dos alternativas. No obstante, no es una tarea sencilla ofrecer una síntesis de los modelos pedagógicos que se utilizan en las instituciones educativas, por cuanto existen configuraciones deducidas a partir de sistemas antropológicos, filosóficos, neuropsicológicos, políticos, sociológicos. Además, tal reto escapa de las pretensiones de este libro, que sólo pretende ayudar a clarificar la diversidad de modelos existentes, su esencia y particularidad, con el fin de que sirva como insumo en el proceso de configuración del modelo pedagógico de la organización educativa. Se pretende contribuir a la capacitación de los profesores para que, desde una posición deontológica, elaboren el modelo propio que sea más pertinente con el proyecto educativo institucional y con su ideal formativo.

Existen diversas tipologías y clasificaciones de los modelos pedagógicos (Álvarez y González, 2003; Flórez, 2005; Peiro, 2005; De Zubiría, M., 2006; De Zubiría, J., 2011; Not, 2013, Hernández, 2014), pero lo más importante para la institución educativa y para el profesor no es el dominio de todas y cada una de estas clasificaciones, sino el análisis crítico de las diversas miradas sobre la educación y la formación del ser humano, para poder asumir una posición armónica y coherente, en correspondencia con su ideal formativo y su contexto sociocultural.

Álvarez y González (2003) advierten que históricamente los modelos pedagógicos se clasifican en dos tipos: instruccional y activista; pero al interior de cada uno de ellos se van 
generando diferencias que generan otros tipos más específicos de modelos, y así el modelo instruccional incluye al modelo tradicional y al modelo conductista, y el modelo activista abarca al modelo desarrollista y al modelo social.

Flórez (2005) deriva los modelos pedagógicos de manera hermenéutica a partir de los postulados pedagógicos de la Escuela Nueva y del concepto de formación. Así, ejemplifica con los modelos pedagógicos romántico, tradicional, desarrollista, conductista y socialista. En una de las instituciones educativas objeto de estudio se visualiza un enfoque tradicional en el aula de jardín ya que la maestra absolutiza el aspecto externo, también se observa una estandarización de la pedagogía en los aspectos de enseñanza, además se aprecia el empleo de métodos autoritarios y directos en el desarrollo de la clase.

Cada modelo pedagógico tiene limitaciones, la Escuela del Desarrollo Integral o Desarrollista, intenta superar diversos aspectos insatisfechos por las teorías que le preceden. Por ejemplo, Peiro (2005) dice que actualmente nos situamos en las perspectivas de cuatro modelos: el modernista-pragmático, el culturalista, el existencialista y el neo-humanista-personalista. Este autor deriva el modelo liberal-clasicista de dos teorías: hermenéutica y análisis-lógico; el modelo cultural-comunitarista emerge de la hermenéutica y de las filosofías de la acción; en virtud de la filosofía analítico-empírica y el instrumentalismo-pragmatista, deviene el modelo progresivista, con dos modalidades: pragmático o dialéctico-materialista; y de la fenomenología y espiritual se configura el modelo esencial-personalista. Asimismo, De Zubiría (2011) hace una clasificación de los modelos pedagógicos de la siguiente manera: heteroestructurante, autoestructurante de la escuela activa, autoestructurante y enfoques constructivistas, y dialogante. Esta taxonomía se sustenta en la tipología establecida por Not
(2013): modelo pedagógico heteroestructurante, modelo pedagógico autoestructurante y la enseñanza dialogante, publicada por vez primera en Francia en el año 1979.

De Zubiría (2006) clasifica los modelos pedagógicos en seis tipos: clásicos, modernos, contemporáneos, funcionales, estructurales cognitivos y estructurales cognitivo-afectivos. Ortiz (2009) destaca que las pedagogías clásicas se subdividen en dos enfoques pedagógicos: tradicional y humanista. Asimismo, las pedagogías modernas se dividen en industrial y activa. Las pedagogías contemporáneas incluyen los enfoques funcional y estructural. Las pedagogías funcionales están representadas en el aprendizaje basado en problemas (ABP). De Zubiría (2006) propone cuatro enfoques pedagógicos que conforman las pedagogías estructurales cognitivas: modificabilidad cognitiva, aprendizaje significativo, pedagogía problémica y cambio conceptual, metodológico, actitudinal y axiológico. Finalmente, este autor clasifica las pedagogías estructurales cognitivoafectivas en dos enfoques pedagógicos: la enseñanza para la comprensión y la pedagogía conceptual, que él configura y desarrolla.

La contraposición que se viene dando desde el siglo XVIII entre dos perspectivas pedagógicas está dada básicamente por la concepción que tiene cada una acerca del estudiante. Una de las propuestas exige que el profesor instruye, enseña y forma. Desde el exterior se dirige al niño, se controla, se modela, se equipa y se moldea. No hay relación sujeto-objeto, sino relación objeto-objeto. Tanto el niño como el contenido de la asignatura que estudia son objetos. La otra perspectiva promulgada por Rousseau (2011) expresa que el estudiante tiene todas las potencialidades para autodesarrollarse, no sólo en la dimensión intelectual sino además en la dimensión moral, por lo tanto, toda acción externa entorpece, bloquea, obstaculiza y deforma su desarrollo natural. 
En el modelo pedagógico tradicional el ser humano se forma (¿o se instruye?) a partir de dos pilares esenciales: la razón y la moral. Se trata de lograr una persona ilustrada según los estándares establecidos por la cultura de la sociedad. Se pretende educar un individuo erudito, compilador del saber, con amplios conocimientos generales y un comportamiento lineal según los cánones establecidos por la iglesia y la sociedad. El proceso educativo se sustenta en la normativa establecida por la sociedad, para educar con disciplina y voluntad en función de formar las virtudes y el carácter. En este modelo la escuela reproduce el estatus quo del mundo de la vida, es la reproducción de la que nos hablan Bourdieu y Passeron (1996) en su teoría del sistema de enseñanza, en la que afirman que ésta contribuye de manera concreta y como ningún otro sistema a la perpetuación de las estructuras de la sociedad, delineando así su tesis central: la autonomía del sistema escolar es relativa. El profesorado y la escuela como sistema son cómplices-víctimas del sistema que reproducen, el mismo sistema que los domina.

En este modelo la escuela a través del profesor inculca al estudiante toda la historia de la cultura societal para que reproduzca el sistema. El profesor es un simple expositor del saber acumulado por la sociedad, se concentra más en la enseñanza, soslayando el aprendizaje, es un instructor, un dictador de clases, un transmisor de conocimientos, reproductor de saberes, una persona rígida, exigente, castigadora, severa, dogmática y autoritaria, que establece una relación vertical con un estudiante dominado, manipulado, un estudiante imitador, repetidor, memorístico, receptivo, vacío de conocimientos y dispuesto a depositar en su cabeza los saberes que el profesor le transmite.

Este modelo pedagógico es caduco y obsoleto, ha llevado a la escuela al fracaso, como lo denuncia Holt (1982), quien devela los excesos y defectos que la mutilan, tales como su afán de competitividad, una didáctica sustentada en monólogos magistrales, un proceso de aprendizaje repetitivo y mecánico, una organización escolar rígida y la proliferación de exámenes descontextualizados y totalmente desvinculados de la vida cotidiana. El modelo pedagógico tradicional es el homicida de la escuela moderna y ha colocado sus principios en su lápida, minando los cimientos de una educación vivencial, auténtica y desarrolladora, para conspirar contra la práctica pedagógica al atentar contra la comunicación y la libertad, que son sus dos grandes fundamentos.

Una denuncia similar hace Laval (2004) al develar los rasgos que caracterizan el ataque neoliberal a la enseñanza pública, agresión que se ha configurado utilizando como cimiento los principios del modelo pedagógico tradicional, haciendo una analogía ilegítima entre la escuela y la empresa, mediante la cual se aniquila la democracia, al no dar cabida a la reflexión pedagógica, reforzando el conformismo, la pasividad y la obediencia, que genera burocratismo y engendra un sistema escolar centralizado, dominante y macabro. Evidentemente la escuela no es una empresa, ni desde el punto de vista ontológico ni desde una mirada teleológica. Incluso, no es una empresa ni desde la óptica epistemológica y mucho menos desde la opción metodológica. Es una tristeza que en Colombia en particular, y en Latinoamérica en general, aún estamos levitando, aletargados por nuestra alucinación.

La escuela pasiva, caracterizada en el modelo pedagógico conductista, tradicional o instruccional tiene como función principal moldear la conducta de los estudiantes según las necesidades sociales. Para lograrlo se formulan objetivos y resultados esperados, se realizan diseños instruccionales y se especifican técnicas concretas para alcanzarlos. En este modelo los trayectos escolares son lineales, controlables, y aparentemente predecibles 
y medibles. Los contenidos de las diversas áreas del saber son abstractos, fragmentados y enciclopédicos. El rol del profesor es secundario y se potencia el empleo de recursos didácticos mediadores, tales como los libros de texto y los proyectores audiovisuales. Las evaluaciones, en correspondencia con las características del proceso educativo, son cuantitativas, reproductivas y memorísticas. Este modelo engendró los exámenes tradicionales con preguntas de selección múltiple con única o múltiples respuestas, y contestaciones de falso o verdadero o completamiento de frases.

Este modelo transmisionista concentra sus esfuerzos más en la enseñanza que en el aprendizaje, por cuanto parte de una concepción externalista que se refiere a la adquisición del conocimiento, no a su configuración por parte del sujeto cognoscente. En este modelo el conocimiento se reduce a datos e informaciones. Es el modelo de la no formación (información), comprendiendo literalmente el prefijo in.

Como se aprecia, la prioridad de la escuela tradicional se enfocó en el mero aprendizaje de conocimientos específicos y normas de conducta muy concretas, la transmisión cultural y que el estudiante imitara y copiara el acervo cultural de las generaciones anteriores, centrándose en la falta de reflexión crítica y creativa, el formalismo, el autoritarismo y el mecanicismo.

A lo largo de la historia de la humanidad, en las prácticas pedagógicas de los profesores, ha predominado la pedagogía tradicional. Como modelo pedagógico no tiene muchos defensores, pero como praxis educativa es aplicable con frecuencia en la cotidianidad pedagógica, aunque en el discurso se hagan férreas críticas. En este modelo el profesor es un sujeto y el estudiante es un objeto. El profesor enseña y el estudiante aprende. El profesor manda y ordena, y el estudiante obedece y ejecuta. El profesor transmite conocimientos, informaciones y normas culturalmente establecidas para que sean asimiladas y apropiadas por el estudiante. El profesor al enseñar, no es considerado activo, porque reproduce los saberes que han sido elaborados por las generaciones anteriores fuera del contexto escolar. Su desempeño exitoso se garantiza depositando informaciones en la cabeza del estudiante y atiborrando su mente de conocimientos con poca pertinencia social. El estudiante al aprender, es pasivo, es un simple receptor de los saberes que transmite su profesor.

La pedagogía tradicional sustentó sus propuestas pedagógicas en los postulados psicológicos de Pavlov (1849-1936), Watson (1878-1958 y Skinner (1904-1995). De esta manera profundizó su concepción memorística y verbalista de la enseñanza, no tenía en cuenta las opiniones, intereses, motivaciones y necesidades de los estudiantes, no promovía el debate, la discusión ni la reflexión. Se convirtió en un modelo educativo autoritario y vertical.

Con mucha frecuencia la escuela tradicional se asocia sólo al método de enseñanza utilizado por el profesor, pero es algo más que eso. Es una forma de pensar, un paradigma mental, una concepción epistemológica, una comprensión externalista del ser humano y su educación. Como afirma De Zubiría (2006), "es una forma de entender los propósitos, los contenidos, la secuencia, la metodología y la evaluación. Es, por ello, un modelo pedagógico que define unas líneas de trabajo y un sentido a la educación" (p. 89).

Por el contrario, la escuela activa promueve que la institución educativa debe ser un espacio agradable para la socialización y el desarrollo personal de los estudiantes. La escuela debe preparar para la vida, pero no en un futuro sino hoy, aquí y ahora, asegurar la felicidad y la libertad de los estudiantes. La escuela nueva postula un aprendizaje activo y participativo, que 
es generado a partir de la actividad, la acción y la experiencia. De esta manera concibe al estudiante como protagonista y actor principal de su propia educación, por lo tanto debe ser considerado el centro del proceso formativo. Desde esta mirada, la escuela nueva se sustenta en la experimentación, la vivencia y la experiencia de los estudiantes.

Es importante destacar que ya desde finales del siglo XIX se fue conformando un movimiento pedagógico extraordinariamente crítico contra el modelo imperante en esa época. Este movimiento se denominó escuela nueva o escuela activa. Aguayo (1933) cita a Ferriere (1910) como el autor que define por vez primera los rasgos esenciales de la escuela nueva, cuyos principales representantes fueron Claparéde (1873 -1940) y Ferriere (1879-1960) en Suiza; Montessori (1870-1952) en Italia; Decroly (1871-1932) en Bélgica; Cousinet (1881-1973) y Freinet (1896-1966) en Francia, y Dewey (18591952) y Kilpatrick (1871-1965) en Estados Unidos, quienes criticaron los postulados de una pedagogía clásica, pasiva o tradicional, y asumieron la defensa del aprendizaje por encima de la enseñanza. Este movimiento pedagógico de rechazo a la escuela industrial, conocido como pedagogía activa proponía orientar a los niños ante la vida y no ante la escuela, abogaba por que la escuela fuera el medio, no el fin. La génesis de este movimiento podemos encontrarla en la obra de Rousseau.

Rousseau (1712-1778) inició en el siglo XVIII lo que podríamos denominar la revolución educativa, en franca protesta en contra del formalismo anacrónico y racionalista que reinaba en esa sociedad. Estremeció con sus ideas naturalistas la concepción de la educación en su época, y nos enseñó a pensar de una manera diferente acerca de la educación. Esbozó nuevos ideales educativos en los que proponía educar con base en los hábitos y disposiciones primarias de los niños, entendiendo que el ser humano es bueno al nacer y que la instrucción de la sociedad es la que distorsiona su conducta. Es por ello que en su extraordinaria obra Emilio o de la Educación (1762) propone una nueva teoría educativa sustentada en una psicología evolutiva. En esta obra sustenta un sistema educativo privado que tiene como finalidad impedir que la sociedad corrompa al joven, de ahí que sólo le permite un contacto limitado con ella a los 20 años de edad. El niño se educa hasta los 12 años de edad casi sin contacto con la civilización.

Estos planteamientos de Rousseau influyen notablemente en su época. A partir de sus postulados se modifica de manera considerable la concepción que se tenía sobre la escuela, el niño y el educador, se incluyen nuevas asignaturas y se reforman los planes de estudio. Sus ideas impregnaron las concepciones de muchos revolucionarios educativos del siglo XIX, pero de manera particular pueden evidenciarse en las obras de Pestalozzi (2011) y Herbart $(1806,1935)$.

Pestalozzi (1746-1827) enfoca su propuesta en la educación moral, sustentado en los postulados teóricos de Rousseau pero precisándolos e integrándolos con nitidez en una dimensión educativa real y práctica con una orientación moderna, incorporando los principios de una pedagogía social que expone en su obra Cómo Gertrudis educa a los niños (1801). Asume que la educación moral se logra al integrar el desarrollo de las facultades físicas, la educación manual y el principio de la intuición como esencia básica de la educación intelectual.

Fróbel (1782-1852) toma de Pestalozzi la concepción de la práctica educativa, integrándola a la concepción idealista de Rousseau sobre la naturaleza humana, y así fundamenta su propuesta basada en el desarrollo espontáneo del potencial interior de los niños. A partir de esta integración desarrolló la noción de educación 
integral, aplicada a los párvulos utilizando materiales concretos, originales y novedosos en los institutos de enseñanza creados por él, a los cuales evitó llamarles escuela porque no le agradaba este término. Estos criterios pedagógicos los validó en sus laboratorios de investigación didáctica, y luego los expuso en su obra La educación del hombre (1826).

La influencia de Fróbel ha llegado hasta nuestros días, expresada en los jardines infantiles (kindergarten), término que ilustra su concepción acerca de la educación infantil. Este tipo de escuela es un jardín en el que cada niño es una flor en crecimiento que debe ser bien cuidada y cultivada para poder disfrutar su color y aroma. En este sentido le da mayor importancia a la actividad del niño que al rol del maestro, éste debe limitarse a seguir de manera natural y espontánea los intereses, motivaciones y necesidades de los niños, para lo cual el juego es la actividad movilizadora del potencial infantil.

Por otro lado, Herbart (1806), al igual que Pestalozzi, postula la moralidad como la finalidad última de la educación, y formula la teoría didáctica de los pasos formales, sustentado en la filosofía y en la psicología, ciencias que le permitieron configurar una pedagogía científica, sistematizando nociones y conceptos universales con el fin de modelar la realidad educativa basándose en estos conceptos.

Ya en el siglo XIX, John Dewey (1859-1952), continúa desarrollando este movimiento pluralista y abierto denominado Escuela Activa o Escuela Nueva, plasmando sus revolucionarias ideas en obras muy significativas: Mi credo pedagógico (1897), La escuela y la sociedad (1899), y El niño y el programa escolar (1902), Democracia y educación (1916). Dewey (1926, 1964, 1989, 1997, 2003, 2004) cuestiona con sólidos argumentos la enseñanza dogmática, formal, rígida y mecánica, crea un nuevo concepto pragmático de educación, proponiendo un aprendizaje basado en el interés y en la acción del niño. En sus obras esboza y sustenta sus concepciones y teorías sobre la importancia de la actividad y la experiencia en la educación del ser humano, situando al estudiante en el centro del proceso de educación. Todo profesor debería leer estas obras por cuanto constituyen referentes importantes para la transformación de nuestras prácticas pedagógicas e insumos epistémicos muy valiosos en el proceso de configuración del modelo pedagógico de la organización educativa. Simultáneamente, Celestín Freinet (1896-1966), propone una serie de métodos y técnicas que buscan activar el rol del estudiante, asignarle un mayor protagonismo y responsabilidad. Debido a esta propuesta, Freinet $(1969,2014)$ integra también el activismo educativo y el movimiento de la Escuela Nueva. En este modelo se inscribe el discurso pedagógico de Montessori (1913), quien consideraba que la educación debe estar encaminada a formar seres humanos libres, democráticos, útiles a la sociedad, para lo cual propone el método clínico de aprendizaje, que consiste en adaptar la enseñanza al aprendizaje y tener en cuenta los estados de salud cognitiva, mental y fisiológica de los niños y niñas.

Estudiando la esencia y naturaleza del modelo de la Escuela Nueva podríamos decir que la educación es auto-reconfiguración, se debe educar partiendo de la realidad, de la experiencia, de las vivencias de los estudiantes y no sólo de los libros, realizando una práctica útil y fecunda, para lo cual es necesario liberar al estudiante de las barreras que entorpecen su desarrollo y quitarles los obstáculos para que aprendan a pensar. Este modelo propone tener en cuenta la necesidad del estudiante, su interés y espontaneidad, en un proceso de libre expresión que forma en la vida, por la vida y para la vida.

En la escuela nueva caracterizada en el modelo pedagógico desarrollista, activo o social los 
estudiantes aprenden haciendo, se privilegia el aprendizaje por encima de la enseñanza y el rol protagónico lo tiene el estudiante, pasando la actividad del profesor a un segundo plano. Los estudiantes aprenden en actividades que tienen en cuenta sus necesidades, deseos e intereses, interactúan con objetos, con el profesor y con sus compañeros en actividades cognoscitivas, praxiológicas, valorativas y comunicativas, que definen la relación sujeto-objeto (actividad) y sujeto-sujeto (comunicación). Se aprende a partir de la experiencia, interactuando con situaciones cotidianas, privilegiando las vivencias de los estudiantes; es un aprendizaje por descubrimiento, significativo, cooperativo y problematizador, cuya intencionalidad es la comprensión, la estimulación del pensamiento crítico, reflexivo y creativo, y la configuración de conocimientos, habilidades, destrezas, actitudes y valores.

Not (2013) precisa que las pedagogías activas rechazan el control, las restricciones, las obligaciones y directivas como métodos de enseñanza y como mecanismos educativos, reivindicando la espontaneidad, la libertad, las necesidades individuales, y sobre todo la "representación más o menos fiel de las concepciones psicoanalíticas, sugieren este rechazo de las restricciones y valoran las situaciones en las que se liberan los procesos creativos que permiten a cada personalidad expresar lo que tiene de original" (p. 136). Dentro de este movimiento podemos percibir diversas tendencias y corrientes incluso divergentes. Por ejemplo, la escuela libertaria Summerhill (Neill, 2010, 2012, 2013) liderada por Alexander Sutherland Neill tenía una concepción muy diferente de la granja Gorki en la antigua Unión Soviética, liderada por Antón Semyonovich Makarenko, en la que se estimulaba el trabajo y el estudio como pilares del proceso formativo.

La Revolución Rusa de 1917 configura una época de promesas en la que la alegría y la felicidad por un maravilloso porvenir se convierten en el tema central de la propuesta pedagógica de Antoni Semyonovich Makarenko (1888-1939), quien desarrolla una novedosa experiencia educativa en la granja Gorki (19201928), publicada posteriormente en el Poema pedagógico, su magna obra. La propuesta educativa de Makarenko está encaminada a cumplir los intereses sociales a partir de las acciones individuales, la naturaleza humana debe adaptarse a las necesidades de la sociedad, para lo cual se debe forjar la voluntad de los jóvenes y ajustar sus intereses individuales a las necesidades colectivas, a partir de una fuerte organización y una férrea disciplina. En esta propuesta la educación se funda en los valores comunistas subordinados al Partido y al Estado, por lo que la felicidad no es individual, se logra en la colectividad, en el cumplimiento de los objetivos sociales comunes, para lo cual se requiere un alto desarrollo de la conciencia colectiva, compromiso, participación, sentido de pertenencia y una alta responsabilidad social. El objetivo de la pedagogía de Makarenko es construir la sociedad comunista, es por ello que educaba en la comunidad y para la comunidad, donde lo individual no sólo es subordinado a lo social sino que es solapado por éste. En cambio, muy diferente a la propuesta de Makarenko, aunque con la misma intencionalidad ideopolítica, Vasil Sujomlinski (1918-1970) considera que la comunidad no es un fin en sí misma sino un medio para formar al hombre comunista. Este personalismo es lograble a través de la acción comunitaria y de la interacción colectiva. Sólo en este ambiente comunitario y en el colectivo laboral en busca del bien común es posible desarrollar todas las potencialidades del ser humano. De esta manera, Sujomlinski utiliza la ideología comunista para matizar su propuesta educativa personalista y romántica. La escuela de la alegría es el nombre que le da a su propuesta pedagógica, que se basa en el niño, en el individuo, al cual le trata de desarrollar 
todas sus potencialidades, mediante la libertad, la felicidad y la alegría.

Sus principales ideas están publicadas en sus codiciadas obras tituladas El mundo espiritual del escolar (1961), La educación de la personalidad en la escuela soviética (1965), Mi corazón es para los niños (1969). Sujomlinski (1975) encamina su acción a cada niño en específico, a la persona en particular, configurando un enfoque personalista y personológico: "Tres mil setecientas páginas suman los cuadernos... cada página está dedicada a una persona, a un alumno mío... cada niño era un mundo, un mundo peculiar, un mundo único" (p. 47).

La escuela pasiva o tradicional puede ser caracterizada como un modelo heteroestructurante. La escuela activa o nueva puede ser valorada como un modelo autoestructurante. Ante estas propuestas se impone un enfoque constructivista, un modelo interestructurante y dialogante que valora el rol activo y protagónico del estudiante en su aprendizaje sin soslayar la importancia de la acción del profesor como mediador de dicho proceso: un modelo que garantiza una síntesis dialéctica (Not, 2013). Este modelo se caracteriza, por una serie de planteamientos, supuestos y opciones básicas con relación al proceso de educabilidad y de enseñabilidad, con una idea integral del desarrollo humano, en cuanto a competencias, habilidades y potencialidades, lo cual expresa de manera resumida en la forma de explicar el ser y el mundo, orientado dentro del proyecto curricular.

En este modelo se trata de entender la educabilidad como un proceso de configuración y reconfiguración personal y la enseñabilidad a partir de la combinación de los saberes previos y nuevos. Los educandos comienzan el proceso de nuevos aprendizajes con una configuración precedente de experiencias y conocimientos que ha configurado a lo largo de la experiencia educativa y lo que se aprende por el hecho de vivir en sociedad (porque no es tabla rasa). Este proceso lo realiza cada educando por medio de un aprendizaje integral relacionado con los saberes previos a través de la reflexión crítica y analítica.

\section{TENDENCIAS PEDAGÓGICAS POSTMODERNAS}

De los modelos pedagógicos y teorías psicológicas derivaron otras tendencias pedagógicas que hoy hacen presencia en los discursos y prácticas pedagógicas. Por ejemplo, la pedagogía conceptual (De Zubiría, 2008), que busca formar un joven amoroso y talentoso. Es preciso destacar que las líneas teóricas más relevantes de la Pedagogía Conceptual constituyen una alternativa a los enfoques clásicos del aprendizaje.

El estudio prolongado de las obras de Piaget $(1945,1954)$ y de Merani condujo a De Zubiría (2006) a concluir que los "conceptos", las "nociones" y las "categorías" ocupaban un lugar privilegiado para comprender el aprendizaje. A todos ellos, genéricamente, los denominó Instrumentos de Conocimiento, sin los cuales el estudiante no puede aprender. De Zubiría y De Zubiría (2002) afirman que la pedagogía conceptual propone como contenidos los instrumentos psicomotrices, cognoscitivos y valorativos

La Pedagogía Conceptual es un enfoque pedagógico holístico que comprende el proceso de aprendizaje no sólo desde la configuración cognitiva e intelectual, sino desde todas las configuraciones de la mente humana: la afectivaemocional (sentir, ser, actitudes, valores, sentimientos y motivaciones); la cognitiva (categorías, conceptos, conocimientos, nociones, saber) y la expresiva o ejecutiva (comunicación, destrezas, habilidades, hacer, prácticas y procesos). "Para ser realmente competentes, frente a cualquier aspecto 
en nuestra vida cotidiana, afectiva, laboral, profesional, académica, debemos querer-saberhacer" (De Zubiría, 2008, p. 19). Sin embargo, De Zubiría (2011) considera que entre los valores y el pensamiento existe una interrelación mucho más profunda y amplia de lo previsto en algunas propuestas pedagógicas innovadoras del siglo XXI como la Pedagogía Conceptual. De esta manera considera que la interdependencia es un concepto central de la Pedagogía Dialogante, es un principio que expresa el carácter integrado de los sucesos, los cuales se escalonan en un proceso interrelacionado. Asimismo, la pedagogía sistémica (Traveset, 2013) es una nueva forma de mirar la educación que genera extraordinarios cambios en nuestra forma de pensar lo educativo y en nuestras actitudes hacia los actores educativos. Desde este enfoque pedagógico, la escuela se orienta hacia el aprendizaje de la vida, con el fin de que los adolescentes y jóvenes hagan algo útil utilizando las enseñanzas de sus padres y demás familiares.

Como se aprecia, constantemente están emergiendo "nuevas" propuestas de enseñanza o modelos educativos, los cuales desconocen sus fundamentos pedagógicos. Esto trae como consecuencia que cada vez que aparece una propuesta con algún aspecto novedoso para la enseñanza y la educación, se piensa que es un nuevo modelo pedagógico totalmente auténtico y original, esto debido a que no se develan las corrientes de pensamiento que configuran la Pedagogía como ciencia, disciplina o saber. Se afirma que dichas propuestas son modelos pedagógicos y en realidad son actualizaciones que incorporan otros aspectos no contemplados por las corrientes o teorías originales del pensamiento pedagógico. Nos estamos refiriendo a la pedagogía conceptual, pedagogía dialogante, pedagogía o escuela transformadora, pedagogía problémica, pedagogía de las competencias, etc. En este sentido, sugerimos conocer los fundamentos de la Pedagogía para poder comprender e interpretar las diversas propuestas didácticas teniendo en cuenta sus cimientos pedagógicos.

También se habla de la «pedagogía por proyectos», tautología muy utilizada en la actualidad, incluso se dice que Finlandia ha eliminado todas las asignaturas del currículum escolar y las ha sustituido por proyectos académicos esenciales y transversales. Toda pedagogía genuina implica a los estudiantes en algún proyecto con determinada finalidad. De manera que la noción de pedagogía de proyectos es un pleonasmo. La pedagogía cuando es legítima organiza al grupo escolar en función de un proyecto y guía a cada estudiante a responsabilizarse con actividades de aprendizaje y formación. Pedagogía de proyectos es una cacofonía.

En el informe Aprender a ser, resultado de un estudio sobre la situación de la educación en el mundo y las posibles estrategias para su desarrollo, Edgar Faure, quien había sido comisionado por la Unesco en el año 1971 para realizar dicha investigación, esbozó algunas ideas que se anticiparon a las propuestas postmodernas sobre la educación desescolarizada (MacLuhan y Carpenter, 1968; Gooddman, 1973; Reimer, 1974; Illich, 1976; Husen, 1978, 1988; Holt, 1982). En efecto, la educación no debe ser sólo responsabilidad de la institución educativa. No obstante, a pesar de estas teorías desescolarizantes, aún sigue predominando la educación escolar, lo cual indica que la escuela aún no ha fracasado y que aún no ha muerto ni morirá jamás, pero para subsistir debe reinventarse, reconfigurarse, resignificarse, de lo contrario, el porvenir de nuestras escuelas, tal como la conocemos actualmente, está en peligro.

Por otro lado, Sáenz (2003) propone una nueva mirada para la pedagogía, que visibiliza problemas y posibilidades ocultas de la 
dimensión formativa, que no han emergido en la práctica pedagógica moderna. Se trata de un esfuerzo disperso que aún es incipiente, con el fin de resistir las tendencias de la cultura dominante, que pueden ser conscientes o inconscientes, según este autor, quien lo ve como un movimiento contractual, y se abstiene de utilizar la noción de «modelo» pedagógico, por el temor de ubicarnos en los límites del control, la predicción y la racionalización, tan nocivos para los procesos educativos. Sin embargo, en este libro el término modelo pedagógico no se utiliza desde esta concepción, sino desde una perspectiva más hermenéutica y emancipatoria. El empoderamiento de los profesores para que sean dueños de su propio proceso de formación y transformación es uno de los atributos del proceso de configuración del modelo pedagógico de la organización educativa, que no debe ser considerado como una tendencia, término inadecuado por cuanto no es un movimiento consolidado desde el discurso ni desde la práctica pedagógica, sin embargo emerge como "fuerza compensatoria de las pedagogías dominantes" (p. 245).

Una pedagogía de los sentimientos y la imaginación como la que quiere proponer Saénz (2003) es aquella que se centra más en las nociones de autoconfiguración, metáfora, símbolo, creatividad e intuición, que en las nociones de racionalidad, verdad y objetividad, es una pedagogía configurativa que no ignora la subjetividad humana, integra el intelecto con la afectividad y le da el lugar que merecen las emociones en el proceso educativo, es una pedagogía del amor (Ortiz, 2014).

\section{PEDAGOGÍA DEL AMOR}

Nosotros los seres humanos estamos muy necesitados de ternura, afecto y cariño, la caricia es esencial y determinante en la dinámica relacional humana, somos seres que necesitamos acariciarnos, pero la caricia implica proximidad, familiaridad, confianza, sosiego, seguridad e intimidad.

"La ternura acoge, cuida, envuelve sin limitar, abre espacios porque amplia la visión; la ternura no exige. En la ternura se está desde sí mismo con el otro, y se acepta al otro como surge en la relación. En nuestra cultura patriarcal, sin embargo, negamos o desvalorizamos la ternura, aunque la necesitemos cotidianamente en nuestro vivir, porque la consideramos debilidad en su apertura visionaria que nos saca de la lucha y la competencia al aceptar la legitimidad del otro o la otra. En nuestra cultura patriarcal vivimos en la lucha y el esfuerzo, y todo debe vivirse en la confrontación continua con dificultades. En nuestra cultura patriarcal no aceptamos lo natural porque ocurre sin esfuerzo, y... si no hay esfuerzo, no hay mérito" (Maturana y Bloch, 1985, p.235).

Martí (1975) afirmaba que sin amor el ser humano no puede vivir, que sin pan uno puede vivir pero sin amor es muy difícil vivir. Afirmaba que al ser tiernos, configuramos la ternura que gozamos nosotros mismos.

Cuán extraordinario es el pensamiento Martí al plantear lo anterior. Realmente, la ternura y el amor son ingredientes aglutinadores del proceso pedagógico. Es por eso que el cariño es la más elocuente de todas las gramáticas, como señalaba Martí (1975).

Existen diferencias sustanciales y apreciables entre el amor y la agresión. En el amor la otra persona surge como un legítimo otro en la convivencia con uno, y en la agresión el otro es negado como una persona legítima en la convivencia con uno. En ambas conductas relacionales el dominio es diferente. En el amor hay aceptación y en la agresión hay negación.

Los seres humanos no pertenecemos a una historia de agresión o de competencia, crecimos y nos desarrollamos en el amor. Es necesario 
que la escuela beneficie y contribuya a la configuración de la experiencia afectiva, volitiva y cognitiva que el niño trae consigo, para que el niño cumpla en su escuela los sueños y las expectativas que tiene sobre ella, con el fin de lograr que el niño y el joven se relacionen adecuadamente con quienes los rodean, respeten los derechos de los demás, se autocontrolen, se respeten a sí mismos como individualidad, y se autoestimen, lo cual es lograble según configuremos nuestra actividad pedagógica diaria. Con lo anterior, el niño y el joven mantendrán el interés por descubrir el sentido y significado de cada hecho y fenómeno de la vida que les rodea y mantendrán además viva la creatividad, la espontaneidad y el talento que demostraron tener en edades tempranas. Como muy bien afirmaba Vygotsky (1979, 1981), la pedagogía se debe orientar no hacia el día de ayer, sino hacía el día de mañana del desarrollo del niño. Sólo así podrá despertar aquellos procesos del desarrollo que se encuentran, en este momento, en la zona del desarrollo próximo, que se conceptualiza como la distancia que hay entre lo que el niño puede hacer por sí solo y lo que puede hacer con ayuda de los demás.

En cada ser humano hay un hombre ideal, es necesario confiar en el niño, en el joven, amarlos, esta idea es precisamente la piedra angular de nuestro enfoque. La vida emocional del niño y del joven es tan importante que lo emocional, lo racional y lo volitivo deben configurarse de manera dialéctica, por cuanto no se genera esta interconexión se limita la validez de la configuración humana.

En el amor no hay exigencias, ni expectativas, ni concesiones, sólo respeto y aceptación por sí mismo, por el otro, la otra o lo otro. El profesor que se respeta a sí mismo y respeta a sus estudiantes, los escucha y puede escucharlos sin temor a desparecer al hacerlo; la profesora que se respeta a sí misma y respeta a sus estudiantes puede realizar un espacio de colaboración con ellos permitiéndoles ser en su plenitud sin negarlos desde su temor o terquedad. El amor es de hecho el fundamento de la dinámica sistémica que configura el espacio en que los niños pueden crecer como personas responsables capaces de aprender cualquier cosa, y colaborar con otros en cualquier actividad porque no temerán desaparecer en la relación con los adultos. Como ya hemos dicho, los niños y niñas no aprenden materias, asignaturas o contenidos programáticos, sino que aprenden la biopraxis humana que vivencian y experimentan con sus profesores y sus maestras. Los sentimientos y emociones, entrelazados con la configuración cognitiva e intelectual, se conviertan en intereses, en deseos de hacer y conocer, en actitudes, valores y convicciones que determinarán el hombre y mujer que queremos formar.

Según Maturana (1999), el educar en la biología del amor es básicamente sencillo: tan sólo tenemos que ser en la biología del amor. Tenemos que ser con los niños bajo nuestro cuidado en educación tal cual somos con nuestros hijos o nuestros amigos, aceptándolos en su legitimidad, incluso si no estamos de acuerdo con ellos. Todo lo que nuestros amigos e hijos hacen es legitimar, incluso cuando objetamos sus acciones o tenemos serias discrepancias con ellos al respecto.

"En la amistad, las discrepancias o desacuerdos son oportunidades para reflexionar en conversaciones en expansión, no ocasiones para mutua negación. A eso se debe que podamos hablar acerca de todo con nuestros amigos. En las amistades no hay demandas, y cuando aparece una, la amistad se termina. Finalmente, hay total confianza mutua y apertura para la colaboración en la amistad porque estamos con nuestros amigos y hacemos cosas con ellos por placer, no por obligación. Amistad es una palabra en nuestra cultura que la mayor parte del tiempo, sin que nos demos cuenta de 
ello, connota la biología del amor" (Maturana, 1999, p.70).

"El amor no es una virtud. En verdad, el amor no es nada especial, es sólo el fundamento de nuestra existencia humana como el tipo de primates que somos como seres humanos" (Maturana, 1999, p.227).

Según Maturana (1999), lo fundamental no está en aprender mucha matemática, mucha biología, o en aprender mucha historia. Tenemos que aprender biología, matemática e historia, para ver el mundo en que vivimos, pero no para lo que vamos a ser o hacer después, sino para hacernos responsables de lo que hacemos ahora en nuestra biopraxis cotidiana, porque lo que vamos a ser y a hacer después surge precisamente de los deseos, del emocionar en el cual hayamos crecido. "No vamos a proteger el medio ambiente porque sabemos biología; lo vamos a proteger porque nos gusta. Porque nos gustan los árboles vamos a hacer algo por lo árboles; porque nos gusta un entorno no dañado, vamos a protegerlo" (Maturana, 1999, p.213).

Por todo lo que hemos expresado, podemos aconsejarles a los profesores que nunca dejen de pasar la oportunidad en su aula o salón de clase para dar un lugar a la poesía, a la música, al relato emotivo, a los más hermosos sentimientos y cualidades humanas que, junto a los conocimientos, contribuirán a hacer de ese niño o niña un hombre culto y libre, y así podrá sonreír cotidianamente ante cualquier actividad, tarea, situación o problema. Nunca dejes de brindarles amor a tus estudiantes. Ahora bien, no obstante a lo que hemos expresado sobre la primacía del amor con relación al conocimiento, pensamos que el papel del conocimiento es central en cualquier nexo afectivo humano, aunque se piense lo contrario. Aunque sea por una potente razón: se ama o se odia el conocimiento de la persona, no a la persona en sí misma. Al amar se ama no al otro individuo -sus reales actos, intenciones, creencias, valores, actitudes, deseos, frustraciones- sino a una configuración teórica y conceptual que configura nuestra mente. Amamos la idea del otro tal como lo configuramos, tal como lo conocemos; no al otro en sí mismo, es decir, que el otro existe psicológicamente como configuración. EI filósofo alemán Kant $(2004,2013)$ nos enseñó que no es posible conocer a la persona en sí misma.

La pedagogía es Amor. $\mathrm{Y}$ lamentablemente muchos profesores no comprenden esto. Aunque a veces no es necesario comprender. Si amas, la comprensión ocupará un segundo plano. ¿Qué sentido tiene comprender las cosas, si no las amas? Por el contrario, si amas, o no necesitas comprensión o hay comprensión de todo. La base de la comprensión es el amor. No hay comprensión sin amor. $Y$ esta concepción es muy importante para el campo científico y educativo, porque el estudiante jamás podrá aprender algo $\sin$ amor.

¿Cómo puede un profesor aprender y enseñar a amar? ¿El amor se aprende? ¿Podemos pedagogizar el amor? En efecto, el profesor debe enseñar a los estudiantes a amar. $Y$ los estudiantes deben aprender a amar. El profesor debe aplicar la Pedagogía del Amor en sus relaciones con los demás. Ese es el camino hacia el éxito pedagógico, ese es el camino hacia la calidad educativa, ese es el camino hacia el aprendizaje significativo, autónomo, auténtico e infinito, ese es el camino hacia la felicidad.

\section{CONCLUSIONES}

Como se aprecia, es extraordinaria la pluralidad de corrientes, tendencias, enfoques y modelos pedagógicos que se pueden clasificar según su intencionalidad formativa o siguiendo múltiples criterios. Habermas (1982) expone que a lo largo de la historia de la humanidad, los seres humanos han desplegado tres tipos de actividades, que 
pueden ser consideradas acciones significativas que caracterizan su hacer. Estas acciones son el trabajo, el lenguaje y la interacción social.

Para cada acción el ser humano muestra un interés cognoscitivo. Para la acción del trabajo el interés es técnico, para la acción del lenguaje el interés es práctico, y para la acción de la interacción social el interés es emancipatorio. Para cada una de estas acciones e intereses cognoscitivos existen diversas ciencias que configuran conocimientos epistémicos. Estas ciencias son las denominadas empíricoanalíticas, histórico-hermenéuticas o sociocríticas, en dependencia del interés cognoscitivo que predomine en su estructura epistémica y en su intencionalidad epistemológica. De esta manera, los enfoques y modelos pedagógicos que han proliferado a lo largo de la historia de la educación pueden catalogarse como modelos explicativos, comprensivos o transformacionales, según la intencionalidad del profesor: explicar, comprender o transformar. Sería interesante que en las reflexiones que hacen los profesores con la finalidad de configurar este componente del modelo pedagógico de la organización educativa se tuvieran en cuenta estas taxonomías, así como las consideró Pasek (2006) en su estudio sobre las prácticas pedagógicas, y así como lo había hecho Grundy (1998) en su estudio sobre el Currículum como producto o praxis. De esta manera, podríamos clasificar los modelos pedagógicos en tres tipos: los modelos explicativos o controladores, los modelos comprensivos-interpretativos y los modelos críticos o emancipatorios.

Como bien puede apreciarse, existe una amplia variedad de teorías del aprendizaje y modelos pedagógicos. No obstante a la existencia de esta gran variedad de modelos y sus taxonomías, la realidad no es tan nítida, no hay sistemas pedagógicos que podamos clasificarlos desde una epistemología cartesiana, por cuanto existen muchísimas interrelaciones entre unos y otros.

Apartirdeloanterior, emergeelamorcomomodelo pedagógico. Los seres humanos generamos en nuestras reflexiones y conversaciones el mundo en que vivimos, es decir, en nuestro lenguajear y emocionar, y nuestra cultura familiar puede ser generada a partir del modo de vivir que vivamos, si logramos que esta cultura y este modo de vivir sean aprendidos, cultivados, consolidados y conservados por nuestros descendientes. Este mundo que configuremos estará signado por el emocionar que guíe nuestra biopraxis. De todas las emociones que podemos vivir, la única que nos puede guiar en el bienestar y la felicidad humana, es el amor; o mejor dicho, el amar.

Amar es el acto que configura lo humano en el vivir, paso a paso, en cada instante y momento de nuestras vidas, hoy, en este presente, en nuestras biopraxis cotidiana, en este suspiro.

\section{REFERENCIAS BIBLIOGRÁFICAS}

Aguayo, A. M. (1933). Filosofía y nuevas orientaciones de la educación. La Habana: Cultural.

Álvarez, C. y González, E. M. (2003). Lecciones de Didáctica General. Bogotá: Magisterio.

Bateson, G. (1987/1972). Pasos hacia una ecología de la mente. Una aproximación revolucionaria a la autocomprensión del hombre. Buenos Aires: Lumen.

Bourdieu, P. y Passeron, J-C. (1996/1977). La reproducción. Elementos para una teoría del sistema de enseñanza. México: Fontamara.

De Zubiría, J. (2011). Los modelos pedagógicos. Hacia una pedagogía dialogante. Bogotá: Magisterio. 
De Zubiría, M. (2006/1998). Pedagogías del siglo XXI: Mentefactos I. El arte de pensar para enseñar y de enseñar para pensar. Bogotá: Fundación Internacional de Pedagogía Conceptual.

De Zubiría, M. (2008). Las teorías de Pedagogía Conceptual. Bogotá: Fundación Internacional de Pedagogía Conceptual.

De Zubiría, M. y De Zubiría, J. (2002/1987). Fundamentos de Pedagogía Conceptual. Bogotá: Plaza y Janes.

Dewey, J. (1926). La escuela y el niño. Madrid: Ediciones de la Lectura.

Dewey, J. (1964). La ciencia de la educación. Buenos Aires: Losada.

Dewey, J. (1989). Cómo pensamos. Barcelona: Paidós.

Dewey, J. (1997). Mi credo pedagógico. León: Universidad de León.

Dewey, J. (2003). Experiencia y educación. Madrid: Biblioteca Nueva.

Dewey, J. (2004/1916). Democracia y educación. Una introducción a la filosofía de la educación. Madrid: Morata.

Flórez, R. (2005/1994). Pedagogía del conocimiento. Bogotá: McGraw-Hill.

Freinet, C. (1969). La psicología sensitiva y la educación. Buenos Aires: Troquel.

Freinet, C. (2014/1970). Técnicas Freinet de la escuela moderna. México: Siglo XXI.

Gooddman, P. (1973). La deseducación obligatoria. Barcelona: Fontanella.
Grundy, S. (1998). Producto o praxis del curriculum. Madrid: Morata.

Habermas, J. (1982/1968). Conocimiento e interés. Madrid: Taurus.

Herbart, J, F. (1806). Pedagogía General derivada del fin de la educación. Madrid: Ediciones de la Lectura.

Herbart, J, F. (1935). Pedagogía General derivada del fin de la educación. Madrid: La Lectura.

Hernández, F. y Sancho, J. M. (1989). Para enseñar no basta con saber la asignatura. Barcelona: Laia.

Hernández, G. (2014/1998). Paradigmas en psicología de la educación. México: Paidós.

Herrera, J.D. (2013). Pensar la educación, hacer investigación. Bogotá: Universidad de la Salle.

Holt, J. (1982). El fracaso de la escuela. Madrid: Alianza.

Husen, T. (1978). La sociedad educativa. Madrid: Anaya.

Husen, T. (1988). Nuevo análisis de la sociedad del aprendizaje. Madrid: Paidós.

Illich, I. (1976). La sociedad desescolarizada. Barcelona: Barral.

Kant, I. (2004/1784). Filosofía de la historia. ¿Qué es la ilustración? La Plata: Terramar.

Kant, I. (2013/1803). Pedagogía. Madrid: Akal.

Laval, Ch. (2004). La escuela no es una empresa. El ataque neoliberal a la enseñanza pública. Barcelona: Paidós. 
MacLuhan, M. y Carpenter, E. (1968). El aula sin muros. Investigaciones sobre técnicas de la comunicación. Barcelona: Cultura Popular.

Martí, J. (1975). Obras Completas. La Habana: Editorial Ciencias Sociales.

Maturana, H. (1999). Transformación en la convivencia. Santiago de Chile: Dolmen.

Maturana, H. y Bloch, S. (1985). Biología del emocionar y Alba Emoting. Santiago: Dolmen.

Montessori, M. (1913). Antropología pedagógica. Barcelona: Araluce.

Neill, A. S. (2010). Hablando sobre Summerhill. México: FCE.

Neill, A. S. (2012/1960). Summerhill. Un punto de vista radical sobre la educación de los niños. México: FCE.

Neill, A. S. (2013/1994). El nuevo Summerhill. México: FCE.

Not, L. (2013/1979). Las pedagogías del conocimiento. México: FCE.

Ortiz, A. (2009). Manual para elaborar el modelo pedagógico de las instituciones educativas. Barranquilla: Antillas.

Ortiz, A. (2014). Pedagogía del Amor y la Felicidad. Bogotá: Ediciones de la U.

Pasek, E. (2006). Reflexiones sobre la docencia: una práctica plena de intereses subyacentes. Educere. 10 (32), marzo 2006, Mérida.

Pestalozzi, J. E. (2011/1801). Cómo Gertrudis enseña a sus hijos. México: Porrúa.
Piaget, J. (1945). El nacimiento de la inteligencia en el niño. Barcelona: Crítica.

Piaget, J. (1954). Inteligencia y afectividad. Buenos Aires: Aique.

Reimer, E. (1974). La escuela ha muerto. Barcelona: Barral.

Rousseau, J.J (2011). Emilio o De la Educación. Madrid: Alianza.

Sáenz, J. (2003). Hacia una pedagogía de la subjetivación. En: Zuluaga, O.L. et. al. Pedagogía y Epistemología. Bogotá: Magisterio.

Santos, M.A. (2012/2000). La escuela que aprende. Madrid: Morata.

Sujomlinski, V. (1975). Pensamiento pedagógico. Moscú: Progreso.

Traveset, M. (2013/2007). La pedagogía sistémica. Fundamentos y práctica. Barcelona: Grao.

Vygotsky, L.S. (1979). El desarrollo de los procesos psicológicos superiores. Barcelona: Crítica.

Vygotsky, L.S. (1981). Pensamiento y Lenguaje. La Habana: Editorial Pueblo y Educación.

Zuluaga, O. L. (1987). Pedagogía e historia. La historicidad de la pedagogía. La enseñanza, un objeto de saber. Bogotá: Foro Nacional por Colombia.

Zuluaga, O. L. (2005). Foucault: una lectura desde la práctica pedagógica. En: Zuluaga, O.L. et. al. Foucault, la pedagogía y la educación. Pensar de otro modo. Bogotá: Magisterio. 